\title{
In connection with identification of VLF emissions before L'Aquila earthquake
}

\author{
M. K. Kachakhidze ${ }^{1}$, Z. A. Kereselidze ${ }^{2}$, N. K. Kachakhidze ${ }^{1}$, G. T. Ramishvili ${ }^{3}$, and V. J. Kukhianidze ${ }^{3}$ \\ ${ }^{1}$ St. Andrew The First-Called Georgian University of The Patriarchy of Georgia, Tbilisi, Georgia \\ ${ }^{2}$ Iv. Javakhishvili Tbilisi State University, M. Nodia Institute of Geophysics, Tbilisi, Georgia \\ ${ }^{3}$ Ilia State University, Tbilisi, Georgia
}

Correspondence to: M. K. Kachakhidze (manana_k@hotmail.com)

Received: 21 September 2011 - Revised: 7 February 2012 - Accepted: 7 February 2012 - Published: 16 April 2012

\begin{abstract}
The present paper deals with an attempt to check the theoretical model of self-generated seismoelectromagnetic oscillations of LAI system on the basis of retrospective data.

Application of the offered simple model enables one to explain qualitatively the mechanism of VLF electromagnetic emission initiated in the process of an earthquake preparation. Besides, the model enables us to associate telluric character geoelectric and geomagnetic perturbations incited by rock polarization and self-generated electromagnetic oscillations of lithosphere-atmosphere system.

L'Aquila earthquake taken as an example to isolate reliably the Earth VLF emission from the magnetospheric electromagnetic emission of the same frequency range, MHD criterion is offered together with geomagnetic activity indexes.

On the basis of the considered three earthquakes, according to the opinion of authors the model of self-generated seismo-electromagnetic oscillations of the LAI system will enable us to approach the problem of resolution of earthquake prediction with certain accuracy.
\end{abstract}

\section{Introduction}

The present study is based mainly on the data of analysis of Abruzzo earthquake, given in special issue of "Natural Hazards and Earth System Sciences" (Contadakis et al., 2010). In this issue there are described all phenomena accompanying L'Aquila catastrophic earthquake occurred on 6 April 2009.

In our paper we aim to check the feasibilities of application of our schematic model (Kachakhidze et al., 2011) with the view of reliability of earthquakes prediction.
According to the offered model, a segment of the Earth where tectonic stress in earthquake preparation period anomalously increases should have positive electric potential towards atmosphere. The presence of such effect is proved in the papers (Bleier et al., 2009; Eftaxias et al., 2009).

It is known that the period of strong earthquake preparation is rather long, although some effects associated with it are manifested only when the tectonic stress value approaches a limit of geological environment solidity.

Abundant laboratory, ground and satellite observations proved that "fracture-induced physical fields allow a realtime monitoring of damage evolution in materials during mechanical loading. When a material is strained, electromagnetic (EM) emissions in a wide frequency spectrum ranging from $\mathrm{kHz}$ to $\mathrm{MHz}$ are produced by opening cracks, which can be considered as so called precursors of general fracture" (Bahat et al., 2005; Eftaxias et al., 2007a, 2009, Hayakawa and Fujinawa, 1994; Hayakawa, 1999; Hayakawa and Molchanov, 2002).

It should be noted that the stressed rock behaves like a stress-EM transformer. These phenomena are detectable both at laboratory and geological scale (Hayakawa and Fujinawa, 1994; Hayakawa, 1999; Hayakawa et al., 1999; Gershenzon and Bambakidis, 2001; Hayakawa and Molchanov, 2002; Bahat et al., 2005; Eftaxias et al., 2007a; Muto et al., 2007; Hadjicontis et al., 2007). Studies on the small (laboratory) scale reveal that the MHz EM radiation appears earlier than the $\mathrm{kHz}$, while the $\mathrm{kHz}$ EM emission is launched from $97 \%$ up to $100 \%$ of the corresponding failure strength (Eftaxias et al., 2002). On the large (geological) scale, intense $\mathrm{MHz}$ and $\mathrm{kHz}$ EM emissions precede EQs that: (i) occurred in land (or near coastline), (ii) were strong (magnitude 6 or larger), or (iii) were shallow (Eftaxias et al., 2002, 
2004, 2006, 2007b; Kapiris et al., 2004a, b; Karamanos et al., 2006). Their lead time ranges from a few days to a few hours. Importantly, the $\mathrm{MHz}$ radiation precedes the $\mathrm{kHz}$ at geophysical scale as well (Eftaxias et al., 2002; Kapiris et al., 2004a; Contoyiannis et al., 2005). Notice that a complete sequence of SES, MHz and $\mathrm{kHz}$ EM anomalies have been observed one after the other in a series of significant EQs that occurred in Greece (Eftaxias et al., 2000, 2002; Karamanos et al., 2006; Papadimitriou et al., 2008). K. Eftaxias notes as well: "We argue that the detected $\mathrm{MHz}$ and $\mathrm{kHz}$ EM radiation anomalies were emitted from the focal area of the L'Aquila EQ" (Eftaxias et al., 2010). Scientists pay attention to the fact that "The time lags between preearthquake EM anomalies and EQs are different for different types of precursors. The remarkable asynchronous appearance of precursors indicates that they refer to different stages of EQ preparation process" (Eftaxias et al., 2010).

It should be mentioned that theoretical model (Kachakhidze et al., 2011) does not contradict the above described observed facts.

\section{Discussion}

\subsection{How strong will the incoming earthquake be}

The formula (1) (Kachakhidze et al., 2011) has been checked up on the basis of retrospective data with the view of experimental evaluation of a theoretical model. The formula connects with each other analytically the main frequency of the observed electromagnetic emission and the linear dimension (the length of the fault) of the emitted body:

$\omega=\beta \frac{c}{l}$

where $\beta$ is the characteristic coefficient of geological medium and it approximately equals to 1 . Of course it should be determined individually for each seismically active region, or for a local segment of lithosphere.

According to formula (1), for instance, when the frequency is $10^{5} \mathrm{~Hz}$, fault length $l$ already equals to $3 \mathrm{~km}$. It is clear that further decrease in frequency will refer to increase of the fault length, and hence to the expected strong earthquake.

Thus, the formula (1) leads us to the purposeful monitoring of electromagnetic emission spectrum that will enable us to follow the process of formation of the main fault.

Our model enables us to describe successively the precursory effects taking the catastrophic earthquake of L'Aquila as an example.

According to observed data of L'Aquila ULF, MHz and $\mathrm{kHz}$ EM anomalies were detected prior to the L'Aquila EQ that occurred on 6 April 2009. The initially emerged $\mathrm{MHz}$ EM emission is thought to be due to the fracture of a highly heterogeneous system that surrounds a family of large highstrength asperities distributed along the fault sustaining the system. The finally emerged strong impulsive $\mathrm{kHz}$ EM radiation is due to the fracture of the asperities themselves (Eftaxias et al., 2010).

It is known that electromagnetic anomalies in $\mathrm{MHz}$ were fixed on 26 March 2009 and 2 April 2009 (Eftaxias et al., 2010). According to our model it implies that in the vicinity of the incoming earthquake focus the formation of cracks was started, the length of which varied within the frames $300 \mathrm{~m}$.

Electromagnetic anomalies were observed in $\mathrm{kHz}$ on 4 April 2009 (Eftaxias et al., 2010) - referring to the fact that the fault length in the focus was already of the kilometer order.

According to our model in the process of cracks uniting and formation of the main fault, the polarization charges which are formed on the fault surface finally are arranged along the main fault. At this moment separate cracks might play the role of thermoionized channels with different electric conductivity.

Total length of a thermoionized channel of high electric conductivity, with the geological point of view, is the main fault length in the focus and its relation to VLF of EM emission is expressed by the formula (1).

According to the offered model, and correspondingly, the formula (1), it is evident that when fracture stops, the emitted EM emission sieges, which is confirmed by experimental data (Eftaxias et al., 2010).

To demonstrate the adequacy of the theoretical model and the experimental data, alongside with the earthquake of Italy, we have considered other cases of earthquakes (China, Haiti):

(1) Wenchuan earthquake (12 May 2008, $M=8.0$, depth $\sim 19 \mathrm{~km}, 31.121^{\circ} \mathrm{N}, 103.367^{\circ} \mathrm{E}$ ).

It is known that the main frequency of the electromagnetic emission prior to the considered earthquake equaled to approximately $1 \mathrm{kHz}$, which, according to the formula (1), when $\beta=1$, corresponds to the fault length $l \approx 300 \mathrm{~km}$ in the earthquake focus. By the use of this parameter we can calculate a theoretical magnitude. With this in view, we used Ulomov's formula (Ulomov, 1993), which is just for $M \geq 5.0$ magnitude earthquakes:

$\lg l=0.6 M-2.5$,

that is

$M \approx 8.1$

which practically coincides with real magnitude of this earthquake (Zhang et al., 2010).

(2) L'Aquila earthquake (6 April 2009, $M=6.3$, depth $\left.\sim 10 \mathrm{~km}, 42.35^{\circ} \mathrm{N}, 13.38^{\circ} \mathrm{E}\right)$.

According to the data available for this earthquake the fault length in the focus $l \approx 15 \mathrm{~km}$ (Cirella et al., 2009). Before the earthquake the main frequency of electromagnetic emission was approximately $20 \mathrm{kHz}$ (Rozhnoi et al., 2009). For this frequency the formula (1) offers the same value of a 
fault length, while the magnitude calculated according to the Ulomov's formula coincides with the value of real magnitude of the earthquake.

(3) Haiti earthquake (12 January 2010, $M=7.0$, depth $\left.\sim 13 \mathrm{~km}, 18.443^{\circ} \mathrm{N}, 72.571^{\circ} \mathrm{W}\right)$.

In case of Haiti earthquake it was impossible to determine the fault length in the focus. It appears that there was a superimposition of a new fault over the old one.

Our model enables us to resolve roughly the inverse problem too. Namely, due to the fact that $M=7.0$ for this earthquake (Athanasiou et al., 2011) according to the Ulomov's formula we'll have:

$l \approx 50 \mathrm{~km}$

which, in case of $\beta=1$, according to the formula (1) will conform to: $\omega \approx 6 \mathrm{kHz}$.

It is known that in this case electromagnetic emission, in frequency range $(0-20 \mathrm{kHz})$, were recorded by the satellite DEMETER, concerning a time period of 100 days before and 50 days after this earthquake.

Thus, we can take about $10 \mathrm{kHz}$ as the main characteristic frequency, for which $l \approx 30 \mathrm{~km}$.

Thus, the offered model enables us to adjust, gradually, step by step, the magnitude of the forecasted earthquake by means of monitoring the electromagnetic emission during the period preceding the earthquake.

\subsection{When will an earthquake occur?}

The model offered by us is just for shallow inland earthquakes of the magnitude $M \geq 5$.0. Pursuant to the Ulomov's formula, for such magnitudes, fault length in earthquake focus is already of a kilometer order.

According to the formula (1) appearance of $\mathrm{kHz}$ in frequency spectrum means that the main fault starts its formation, but future frequency decrease refers to the increase of incoming earthquake magnitude.

It should be stated that in the first approximation, the analysis of the experimental data proves our theoretical conclusions (Kachakhidze et al., 2011). It is also known from the experiments that strong earthquakes occur in one or two days after $\mathrm{kHz}$ appearance in electromagnetic emission (Eftaxias et al., 2010; Papadopoulos et al., 2010).

Generally, $\omega(\beta)$ frequency of emission depends on the properties of a geological medium. Therefore, if we know the characteristic $\omega(\beta)$ frequency for concrete seismic region, determined on the basis of the retrospective data, the electromagnetic emission monitoring in the period preceding the earthquake will enable us to forecast the time of earthquake occurrence with certain accuracy.

\subsection{Where will an earthquake occur?}

According to our model, it is possible to detect the territory on the surface of the Earth in advance, where an earthquake is expected - the epicentral area of a future earthquake will be approximately limited to the territory where the earth surface will have positive potential towards atmosphere. Electromagnetic emission in $\mathrm{kHz}$ should take place namely on the territory adjoining the epicenter of a future strong earthquake. Our opinions have been experimentally confirmed by the paper of K. Eftaxias (Eftaxias et al., 2010).

Additionally, papers were published in Special Issue (Contadakis et al., 2010) in which various researchers consider the problem of a location of an incoming earthquake on the basis of experimental data (Rozhnoi et al., 2009; Biagi et al., 2009). These works are in good conformity with our theoretical model.

\subsection{Telluric effects of rock depth polarization}

Main fault in the earthquake focus along which a polarization effect should be developed is always of depth character.

According to our model the lower virtual conductor of the hypothetic electromagnetic circuit is located on the Earth surface. For substantiation of justness of such assumption the scheme of prolongation of depth polarized conductor directed towards the Earth surface is rather convenient, which gives simply the vertical electric cross section of the Earth (Kachakhidze et al., 2011).

It should be stated that to construe a model electro dynamic figure corresponding to this section, we have just to allow that the specific electric conductivity $\sigma$ of the Earth from the fault level to the Earth surface decreases. Polarized charge density $\rho$ accumulated here attenuates in time. Allowing such assumption the changes of these parameters might be characterized according to exponential laws

$$
\begin{aligned}
& \sigma=\sigma_{0} e^{-k z}, \\
& \rho(t)=\rho_{0} e^{-\frac{t}{t_{0}}}
\end{aligned}
$$

where $\sigma_{0}$ and $\rho_{0}$ are characteristic values, $Z$ - is the vertical coordinate, $k$ and $t_{0}$ - logarithm decrements of attenuation in space and time.

At the application of these simple models in the equation of electric field potential, it turned out that the polarized linear conductor postulated on the Earth surface qualitatively might be a reflection of a depth fault. We consider that these models, alongside with the electromagnetic circuit of LAI system might be used also in qualitative analysis of electromagnetic perturbations accompanying telluric electric currents.

It is known that anomalous geomagnetic and geoelectric variations (pulsations) have been fixed many times in the periods of earthquake preparation (Varotsos et al., 2009). Difference in their parameters can be connected with changes of electromagnetic characteristics of the Earth and circumterrestrial space. Namely, anomalous increase of ionospheric magnetic viscosity above the focus of incoming earthquake might determine the geomagnetic pulsation frequency spectrum of ionospheric genesis (Kereselidze et al., 


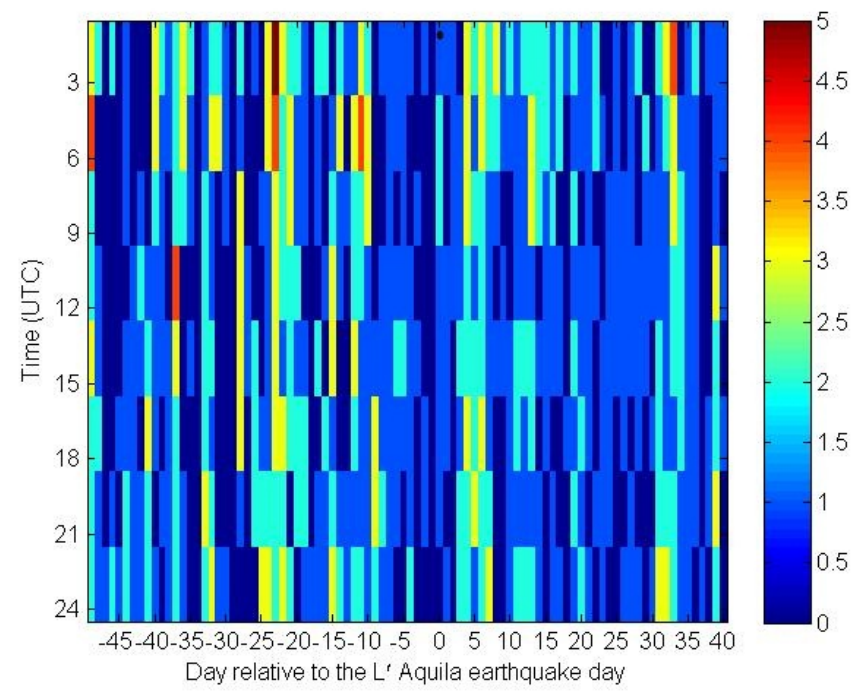

Fig. 1. Changes of $K_{p}$ index in time interval corresponding to L'Aquila earthquake.

2010). Therefore, in spite of the fact that the available data don't enable us to use telluric electromagnetic parameters as earthquake indicators yet, morphological analysis of their changes might appear useful with the view of seismic prediction problem. Thus, appearance of ULF emission before L'Aquila earthquake, on 29 March 2009 (Papadopoulos et al., 2010), can imply that perturbations of telluric currents, with their accompanying effects, had occurred already in the region.

\subsection{Magnetospheric VLF electromagnetic emission}

Usually, such emissions always accompany the increase of solar wind gasdynamics pressure. The same effect occurs in the case of connection of the interplanetary magnetic field on the magnetosphere day-side and the geomagnetic field border force line as well.

This effect, as a rule, incites perturbation of inner structures of the magnetosphere and often it is crowned with the global geomagnetic storm. At this moment the Earth VLF emission will surely be overlapped by a far more intensive magnetospheric emission (Akhoondzadeh et al., 2009). Therefore, in the seismically active middle latitudinal belt the Earth VLF electromagnetic emission should be reliably isolated from the magnetospheric VLF emission of the same frequency range. Namely, in the process of analysis of the Earth VLF emission nobody supposed a possibility of generation of magnetospheric VLF electromagnetic emission in calm, or less perturbed geomagnetic conditions. It is quite natural that application of Earth VLF emission for the purposes of earthquake prediction would not be correct without clarification of a problem.

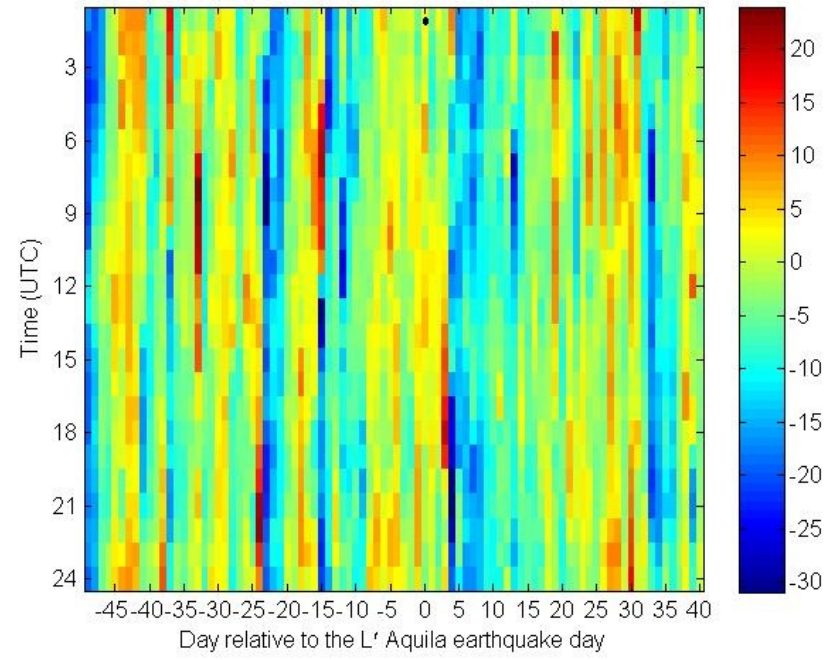

Fig. 2. Changes of $D_{\text {st }}$ index in time interval corresponding to L'Aquila earthquake.

There is a model (Kereselidze et al., 1993), which aims to demonstrate the possibility of generation of VLF electromagnetic emission in the middle latitudes, at the calm and less perturbed geomagnetic conditions. Fundamental basis of this diagnostic model is the classical analytical solution obtained by S. Chapligin, which is just for ideal, incompressible liquid stream approximation. Application of this solution will enable one to simulate the process of flow of the magnetosphere dayside by solar wind (Aburdjania et al., 2007). Large-scale hydrodynamic picture corresponding to it contains the so-called stagnation zone. Maximum dimension of this formation is conditioned by the thickness of the magnetosheath preceding the magnetosphere and the speed of solar wind in interplanetary space.

It is considered that at certain conditions, even at less perturbed solar wind the large-scale laminar stream of solar wind plasma in the magnetosheath preceding dayside of magnetosphere passes to turbulent one. The magneto hydrodynamic criterion of the maximal size stagnation zone border stability can be used as an indicator of changes of magnetosphere flow regime

$M=1-v_{1} / 2 v_{\mathrm{a}} \geq 0$

where $v_{1}, v_{\mathrm{a}}$ - are the solar wind speed and Alphen speed in plasma at the border of stagnation zone correspondingly.

Methodology of calculation of (4) criterion for maximal size stagnation zone is rather simple (Kereselidze et al., 1993), and the necessary data for any moment are accessible on the corresponding cites (http://swdcdb.kugi.koyotee. ac.jp).

According to formula (4), the mark "plus" corresponds to a large-scale laminar regime of magnetosphere flow, while "minus" - to a turbulent one. In the last case the number of 


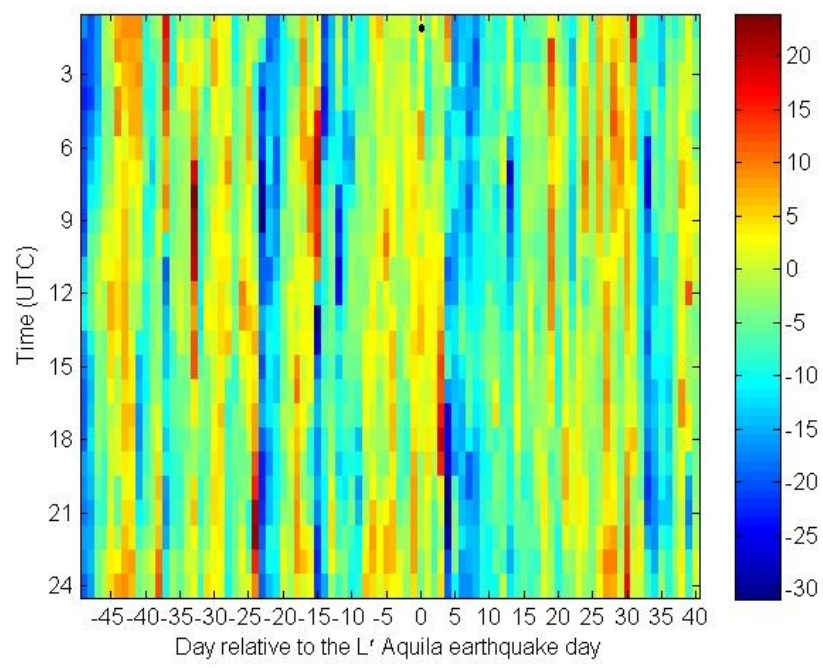

Fig. 3. Changes of $M$ index in time interval corresponding to L'Aquila earthquake.

solar wind energetic electrons, which enter magnetosphere from the bottom of the stagnation zone, might suffer increase, and their stream might contribute to initiation of electron cyclotron instability in plasmosphere (ionosphere) captured plasma and to the generation of the VLF electromagnetic emission. This idea was checked rather reliably some time ago (Kereselidze et al., 1993). It turned out that in calm and less perturbed geomagnetic conditions there the morphological relation really exists between the magnetosphere flow regime and magnetospheric VLF electromagnetic emission. The middle latitudinal VLF emission data were used at the level of upper ionosphere together with the indexes indicating the level of geomagnetic activity. Global middle latitudinal geomagnetic index satisfies the condition $\mathrm{D}_{\mathrm{st}} \geq-15 \mathrm{nT}$ (nanotesla), while the global magnetic activity index $K_{p} \leq 2$ corresponds with the sufficient reserve, to the calm or less perturbed magnetosphere.

The analysis showed that when $M \geq 0$, that is, when according to diagnostic model the solar wind flow large-scale structure near the magnetosphere border had to be laminar, the VLF emission was not fixed practically. Instead, VLF emission was almost always fixed when $M<0$, that is, probably at the turbulent regime of solar wind flow. Aggregate prediction accuracy of these results was rather high $(\approx 85 \%)$. Additionally, further studies revealed that at the increase of latitudinal interval the diagnostic possibilities of the given model decrease. This fact once more refers that plasmosphere should be the source of the magnetospheric VLF electromagnetic emission (Nishida, 1978). Thus, we consider rather reasonable that estimation of geomagnetic activity by $K_{p}$ and $\mathrm{D}_{\mathrm{st}}$ indexes (Akhoondzadeh et al., 2010) is not sufficient for the exclusion of the possibilities of generation of magnetospheric VLF emission. With this in view, application of additional $M$ criterion (index) makes rather strict the demand of geomagnetic indexes, which is proved by the below given example.

Figures 1-3 offer dynamic picture of changes of the $K_{p}$, $\mathrm{D}_{\text {st }}$ indixes and $M$ criterion in three month interval covering L'Aquila earthquake. Geomagnetic perturbation level is limited to the values $\mathrm{D}_{\mathrm{st}} \geq-15 \mathrm{nT}$ and $K_{p} \leq 2$ (on figures the moment of earthquake occurring is marked by a black dot). We have to state that the Fig. 1 practically coincides with the data of the above paper (Akhoondzadeh et al., 2010) (where for $D_{\text {st }}$ index a relatively less strict term is used: $\mathrm{D}_{\mathrm{st}} \geq-20 \mathrm{nT}$ ), while the picture corresponding to $K_{p}$ is identical with our picture.

Simple analysis shows that often, when according to $K_{p}$, $\mathrm{D}_{\mathrm{st}}$ indexes magnetosphere might be considered calm or less perturbed, $M$ criterion is negative. It implied that the magnetospheric VLF electromagnetic emission may exist at middle or lower latitudes.

\section{Conclusions}

The model of self-generated seismo-electromagnetic oscillations of the LAI system somewhat simplifies consideration of various problems associated with earthquakes:

1. For reliable separation of the Earth VLF electromagnetic emission in the seismic active middle latitudinal belt from the same frequency range magnetospheric electromagnetic emission, the special MHD criterion has been offered.

2. In case of electromagnetic emission spectrum monitoring in the period that precedes earthquake it is possible to determine, with certain accuracy, the time, location and magnitude of an incoming earthquake simultaneously.

Acknowledgements. The authors express their thanks to P. F. Biagi and the Anonymous referee for useful notes.

Edited by: M. E. Contadakis

Reviewed by: P. F. Biagi and another anonymous referee

\section{References}

Aburdjania, G. D., Kereselidze, Z. A., Khantadze, A. G., and Chkhitunidze, M. S.: Large-scale LF electromagnetic waves in the Earth's magnetosheath, Geomagn. Aeron., 47, 548-554, 2007.

Akhoondzadeh, M., Parrot, M., and Saradjian, M. R.: Investigation of VLF and HF waves showing seismo-ionospheric anomalies induced by the 29 September 2009 Samoa earthquake $\left(M_{\mathrm{W}}=8.1\right)$, Nat. Hazards Earth Syst. Sci., 10, 1061-1067, doi:10.5194/nhess-10-1061-2010, 2010.

Akhoondzadeh, M., Parrot, M., and Saradjian, M. R.: Electron and ion density variations before strong earthquakes $(M>6.0)$ using DEMETER and GPS data, Nat. Hazards Earth Syst. Sci., 10, 7 18, doi:10.5194/nhess-10-7-2010, 2010. 
Athanasiou, M. A., Anagnostopoulos, G. C., Iliopoulos, A. C., Pavlos, G. P., and David, C. N.: Enhanced ULF radiation observed by DEMETER two months around the strong 2010 Haiti earthquake, Nat. Hazards Earth Syst. Sci., 11, 1091-1098, doi:10.5194/nhess-11-1091-2011, 2011.

Biagi, P. F., Castellana, L., Maggipinto, T., Loiacono, D., Schiavulli, L., Ligonzo, T., Fiore, M., Suciu, E., and Ermini, A.: A pre seismic radio anomaly revealed in the area where the Abruzzo earthquake $(M=6.3)$ occurred on 6 April 2009, Nat. Hazards Earth Syst. Sci., 9, 1551-1556, doi:10.5194/nhess-91551-2009, 2009.

Bahat, D., Rabinovitch, A., and Frid, V.: Tensile fracturing in rocks: Tectonofractographic and Electromagnetic Radiation Methods, Springer Verlag, Berlin, 570 pp., 2005.

Bleier, T., Dunson, C., Maniscalco, M., Bryant, N., Bambery, R., and Freund, F.: Investigation of ULF magnetic pulsations, air conductivity changes, and infra red signatures associated with the 30 October Alum Rock M5.4 earthquake, Nat. Hazards Earth Syst. Sci., 9, 585-603, doi:10.5194/nhess-9-585-2009, 2009.

Cirella, S., Piatanesi, A., Cocco, M., Tinti, E., Seognamiglio, L., Michelini, A., Lomax, A., and Boschi, E.: Rupture history of the 2009 L'Aquila (Italy) earthquake from non-linear joint inversion of strong motion and GPS data, Geophys. Res. Lett., 36, L19304, doi:10.1029/2009GL039795, 2009.

Contadakis, M. E., Biagi, P. F., and Hayakawa, M. (Eds.): Ground and satellite based observations during the time of the Abruzzo earthquake, Special Issue, Nat. Hazards Earth Syst. Sci., http://www.nat-hazards-earth-syst-sci.net/special_ issue 102.html, 2010.

Contoyiannis, Y., Kapiris, P., and Eftaxias, K.: Monitoring of a preseismic phase from its electromagnetic precursors, Phys. Rev. E, $71,1-14,2005$.

Eftaxias, K., Kopanas, J., Bogris, N., Kapiris, K., Antonopoulos, G., and Varotsos, P.: Detection of electromagnetic earthquake precursory signals in Greece, P. Jpn. Acad. B.-Phys., 76, 45-50, 2000.

Eftaxias, K., Kapiris, P., Dologlou, E., Kopanas, J., Bogris, N., Antonopoulos, G., Peratzakis, A., and Hadjicontis, V.: EM anomalies before the Kozani earthquake: A study of their behaviour through laboratory experiments, Geophys. Res. Lett., 29, 69/1-69/4, 2002.

Eftaxias, K., Frangos, P., Kapiris, P., Polygiannakis, J., Kopanas, J., Peratzakis, A., Skountzos, P., and Jaggard, D.: Review and a Model of Pre-Seismic electromagnetic emissions in terms of fractal electrodynamics, Fractals, 12, 243-273, 2004.

Eftaxias, K., Sgrigna, V., and Chelidze, T.: Mechanical and Electromagnetic Phenomena Accompanying Preseismic Deformation: from Laboratory to Geophysical Scale, Tectonophysics, 431, 1301, 2007a.

Eftaxias, K., Panin, V., and Deryugin, Y.: Evolution EM-signals before earthquake and during laboratory test of rocks, Tectonophysics, 431, 273-300, 2007b.

Eftaxias, K., Athanasopoulou, L., Balasis, G., Kalimeri, M., Nikolopoulos, S., Contoyiannis, Y., Kopanas, J., Antonopoulos, G., and Nomicos, C.: Unfolding the procedure of characterizing recorded ultra low frequency, $\mathrm{kHZ}$ and $\mathrm{MHz}$ electromagetic anomalies prior to the L'Aquila earthquake as preseismic ones - Part 1, Nat. Hazards Earth Syst. Sci., 9, 19531971, doi:10.5194/nhess-9-1953-2009, 2009.
Eftaxias, K., Balasis, G., Contoyiannis, Y., Papadimitriou, C., Kalimeri, M., Athanasopoulou, L., Nikolopoulos, S., Kopanas, J., Antonopoulos, G., and Nomicos, C.: Unfolding the procedure of characterizing recorded ultra low frequency, $\mathrm{kHZ}$ and $\mathrm{MHz}$ electromagnetic anomalies prior to the L'Aquila earthquake as pre-seismic ones - Part 2, Nat. Hazards Earth Syst. Sci., 10, 275294, doi:10.5194/nhess-10-275-2010, 2010.

Eftaxias, K. A., Kapiris, P. G., Balasis, G. T., Peratzakis, A., Karamanos, K., Kopanas, J., Antonopoulos, G., and Nomicos, K. D.: Unified approach to catastrophic events: from the normal state to geological or biological shock in terms of spectral fractal and nonlinear analysis, Nat. Hazards Earth Syst. Sci., 6, 205-228, doi:10.5194/nhess-6-205-2006, 2006.

Gershenzon, N. and Bambakidis, G.: Modelling of seismoelectromagnetic phenomena, Russian Journal of Earth Sciences, 3, 247275, 2001.

Hadjicontis, V., Mavromatou, C., Antsyngina, T., and Chisko, K.: Mechanism of electromagnetic emission in plastically deformed ionic crystals, Phys. Rev. B, 76, 024106, doi:10.1103/PhysRevB.76.024106, 2007.

Hayakawa, M.: Atmospheric and Ionospheric Electromagnetic Phenomena Associated with Earthquakes, Terrapub, Tokyo, 1999.

Hayakawa, M. and Fujinawa, Y.: Electromagnetic Phenomena Related to Earthquake Prediction, Terrapub, Tokyo, 1994.

Hayakawa, M. and Molchanov, O.: Seismo Electromagnetics, Terrapub, Tokyo, 2002.

Hayakawa, M., Itoh, T., and Smirnova, N.: Fractal analysis of ULF geomagnetic data associated with the Guam earthquake on August 8, 1993, Geophys. Res. Lett., 26, 2797-2800, 1999.

Kachakhidze, M. K., Kereselidze, Z. A., and Kachakhidze, N. K.: The model of self-generated seismo-electromagnetic oscillations of the LAI system, Solid Earth, 2, 17-23, doi:10.5194/se-2-172011, 2011.

Kapiris, P., Eftaxias, K., and Chelidze, T.: The electromagnetic signature of prefracture criticality in heterogeneous media, Phys. Rev. Lett., 92, 065702, doi:10.1103/PhysRevLett.92.065702, 2004a.

Karamanos, K., Dakopoulos, D., Aloupis, K., Peratzakis, A., Athanasopoulou, L., Nikolopoulos, S., Kapiris, P., and Eftaxias, K.: Study of pre-seismic electromagnetic signals in terms of complexity, Phys. Rev. E, 74, 016104, doi:10.1103/PhysRevE.74.016104, 2006.

Kereselidze, Z., Kachakhidze, M., Kachakhidze, N., and Kirtskhalia, V.: Model of Geomagnetic Field Pulsations before Earthquakes Occurring, Nova Science Publishers Georgian International Journal of Sciences and Technology, Vol. 2, 1-12, 2010.

Kereselidze, Z. A. and Orvelashvili, V. Sh.: Relation of the Solar Wind MHD Flow in the Transition Region to VLF Radiation in the Earth Magnetosphere, Geomagn. Aeronomy, 33, 165-167, 1993.

Muto, J., Nagahama, H., Miura, T., and Arakawa, I.: Frictional discharge at fault asperities: origin of fractal seismoelectromagnetic radiation, Tectonophysics, 431, 113-122, 2007.

Nishida, A.: Geomagnetic Diagnosis of the Magnetosphere, Springer-Verlag, New yark-Heidelberg-Berlin, 1978.

Papadimitriou, C., Kalimeri, M., and Eftaxias, K.: Nonextensivity and universality in the earthquake preparation process, Phys. Rev. E., 77, 036101, doi:10.1103/PhysRevE.77.036101, 2008. 
Papadopoulos, G. A., Charalampakis, M., Fokaefs, A., and Minadakis, G.: Strong foreshock signal preceding the L'Aquila (Italy) earthquake $\left(M_{\mathrm{w}} 6.3\right)$ of 6 April 2009, Nat. Hazards Earth Syst. Sci., 10, 19-24, doi:10.5194/nhess-10-19-2010, 2010.

Rozhnoi, A., Solovieva, M., Molchanov, O., Schwingenschuh, K., Boudjada, M., Biagi, P. F., Maggipinto, T., Castellana, L., Ermini, A., and Hayakawa, M.: Anomalies in VLF radio signals prior the Abruzzo earthquake $(M=6.3)$ on 6 April 2009, Nat. Hazards Earth Syst. Sci., 9, 1727-1732, doi:10.5194/nhess-91727-2009, 2009.
Ulomov, V. I.: Ordering of geostructure and seismicity in seismoactive regions. Seismisity and seismic zoning of Northern Eurasia, Moscow, Vol. 1, 27-31, 1993.

Varotsos, P. A., Sarlis, N. V., and Skordas, E. S. Detrended fluctuation analysis of the magnetic and electric field variations that precede rupture, Chaos, 19, 023114, doi:10.1063/1.3130931, 2009.

Zhang, X., Shen, X., Liu, J., and Ouyang, X.: Ionospheric perturbations of electron density before the Wenchuan Earthquake, Int. J. Remote Sens, 31, 3559-3569, 2010. 